\section{Capgras' Syndrome}

SiR: Gibbs \& Andrewes (Journal, December 1988, $153,853)$ contribute further to the correspondence arising from my paper (Journal, July 1988, 153, 117118 ), in raising the importance of distinguishing between delusional misidentification and prosopagnosia. They advance the possibility that Capgras' syndrome and prosopagnosia reflect a similar, or the same, underlying process and comment, quite correctly, that a distinction between the two is that Capgras' syndrome is considered to represent delusional misidentification of familiar others. However, they feel that, "this cannot necessarily be claimed in Dr Lipkin's case, as the presence of an organicallybased dementia was established".

While welcoming further interest in these matters, I must ask why Drs Gibbs \& Andrewes suggest that the delusional nature of my patient's misperceptions is inconsistent with the presence of her organicallybased dementia. (This patient experienced delusional misidentification, not "delusional" in quotation marks as per their letter). There are many organic/ systemic conditions in which delusional phenomena can arise and indeed delusional Capgras misidentification also, as mentioned in my original paper.

Drs Gibbs \& Andrewes also say in distinguishing between prosopagnosia and delusional misidentification that, "When a labelled photograph of the misidentified individual is provided, prosopagnostics benefit from the label, while individuals with Capgras' syndrome would presumably fail to continue to recognise the individual". My original paper did not mention that this particular patient had indeed manifested delusional misidentification of photographs of her second husband and had not in any way, even before the more florid aspects of her dementing process were in evidence, obtained, as Drs Gibbs \& Andrewes put it, "benefit from the label" (of photographs of her husband). Her husband related to me that she had actually torn up several photographs of him which had been on her dressing table at home, saying that they were not of him, but of an identical imposter, even though other people (whom she seemingly trusted and did not misidentify) had told her otherwise.

Grovelands Priory Hospital

BRON LIPKIN

The Bourne

Southgate

London N14 6RA

\section{Unitary Models of Schizophrenia}

SIR: I read with interest the article by Frith \& Done (Journal, October 1988, 153, 437-443). About three years ago I formulated a unitary view on the aetiology of schizophrenia (Journal, August 1986, 149, 245). Briefly, I thought that some individuals are genetically determined to have weak and vulnerable dopaminergic systems. When subjected to stresses, some of the neurons on which dopaminergic afferents make synaptic contact cease to function. To compensate for the loss, the dopaminergic fibres give off additional nerve terminals and form new D2 receptors. This overreaction gives rise to a relative increase in dopaminergic activity, and manifests itself as the positive symptoms of schizophrenia. In mild cases, the dysfunction is self-limiting and the dopaminergic transmission is able to return to its previous level of functioning, and the process is completed. This psychotic episode is named a schizophreniform reaction. In more severe cases, the dysfunction becomes chronic and progressive. It results in the actual degeneration of the dopaminergic pathways. Negative symptoms occur when the degeneration becomes extensive and when the mesocortical dopamine system is involved. But then how could this cessation of neuronal function and actual degeneration have occurred? The recent studies on the NMDA receptors may provide some clues.

The excitatory amino acids (glutamate, aspartate) act on the NMDA and non-NMDA (kainate, quisqualate) receptors. The non-NMDA receptors, when stimulated, generate fast depolarising responses until a certain level of membrane potential is reached, and then the NMDA receptors are activated. The NMDA-induced current is longer in duration, and $\mathrm{Ca}^{2+}$ ions enter along with $\mathrm{Na}^{+}$through the ion channels. Activity of the NMDA receptors are found to be responsible for plasticity in the developing and mature CNS and long-term potentiation (LTP) in the hippocampus. In the event of prolonged and excessive stimulation, excitotoxicity comes into play when there is too much glutamate and too much calcium. It results in nerve cell damage and death. When the neurons are briefly exposed to glutamate, the cells swell transiently and then go on to recover, but when influx of calcium also occurs, the cells slowly degenerate. This excitotoxicity is postulated to be the cause of degeneration in cerebral hypoxia and ischaemia and in some neurodegenerative disorders such as Huntington's disease and Alzheimer's disease.

The excitatory amino acids are major transmitters of cortico-cortical, corticofugal and sensory systems. Corticofugal pathways run from the cortex to the basal ganglia (nucleus accumbens and caudate/putamen), the hippocampus and the dorsal lateral geniculate, the parts of the brain where highest level of NMDA receptors are found. The striatal NMDA receptors are located on the heads of the dendritic 
spines of the striatal nigral projection neurons. Synaptic transmission in these pathways is blocked by glutamate antagonists. The non-competitive antagonist of NMDA receptors which act at the receptor-operated ion channels is particularly interesting, because it includes phencyclidine and chlorpromazine, which have psychotomimetic and antipsychotic properties respectively.

It is possible that during severe psychosocial stresses the excitatory amino acid pathways are overstimulated, which leads to an increase of glutamate transmission and calcium influx. This results in excitotoxicity effect on the striatal neurons where NMDA receptors are located. This in turn activates the dopaminergic pathways which synapse on the dendritic neck of the same neurons. This overreaction and degeneration lead to the schizophreniform reaction and chronic schizophrenic process as mentioned earlier, depending on the severity of the damage done and the vulnerability of the dopamine system.

According to this hypothesis, NMDA antagonists and calcium channel antagonists may be effective in preventing the progression from acute to chronic schizophrenia. Yet non-competitive NMDA antagonists such as MK 801 and phencyclidine are found to have psychotomimetic effects. The possible reason is that when the NMDA ion channels are blocked, there is increased transmission through the nonNMDA receptors as well as activation of dopaminecontaining elements in the striatum. More research in this area is awaited.

Kowloon Hospital

C. W. Lo

Psychiatric Unit

147 A Argyle Street

Kowloon

Hong Kong

\section{References}

CHOI, D. W. (1988) Calcium mediated neurotoxicity: relationship to specific channel types and role in ischaemic damage. Trends in Neuroscience, 11, 465-469.

Cotman, C. W., Monaghan, D. T., Ottersen, O. P. et al (1987) Anatomical organisation of excitatory amino acid receptors and their pathways. Trends in Neuroscience, 10, 273-280.

KeMP, J. A., Foster, A. C. \& WoNG, E. H. F. (1987) Non-competitive antagonists of excitatory amino acid receptors. Trends in Neuroscience, 10, 294-298.

MARTIN, J. B. \& GUSELLA, J. F. (1986) Huntington's disease; pathogenesis and management. New England Journal of Medicine, 315, 1267-1276.

SIR: Frith \& Done (Journal, October 1988, 153, 437443) provide an elegant account of the symptoms of schizophrenia in relationship to the self-monitoring of actions. The positive symptoms are regarded as a failure of monitoring, because of the degradation of information about willed intentions. In contrast, negative symptoms are associated with a difficulty in forming willed intentions. I would suggest that such an explanation is both faulty and unneccesarily complicated.

The concepts of 'the will' and 'intention', and the associated 'act', are notoriously problematic. (What is an 'act of will' in this context?) Nevertheless, if we in some form accept the existence of 'willed intent' we readily recognise Drs Frith \& Done's suggestion that this in some way fails in the schizophrenic with negative symptoms. However, does it not fail in all of his actions, whether generated from stimuli or from a willed intent? The inability of social stimuli to generate 'normal' and acceptable behaviour is a familiar problem with such patients. Furthermore, we know that the acutely psychotic patient can be disinhibited and also fail to respond to social mores. We therefore have to elaborate the theory, perhaps by arguing that such patients are unable to understand these stimuli. Alternatively, we can make the much simpler statement that all schizophrenics have potential difficulty in forming 'willed intentions' for all kinds of acts. Or should we merely reassert Bleuler's contention that ambivalence of the will is a fundamental symptom of schizophrenia?

Considering now the positive symptoms, the theory has the advantage of describing percepts, beliefs, and emotions as irreducible mental events. "First rank symptoms are essentially abnormal experiences and do not necessarily result in obvious changes in behaviour." It thus becomes easier to understand the fact that a psychotic patient can experience such phenomena at the same time as experiencing both the real world and other people's reactions to him; and that he can use any combination of phenomena to inform his actions. Furthermore, 'insight' can also be seen as a process separate to this event. It can be understood within the context of Drs Frith \& Done's theory as the appraisal of the correlation of this mental event with reality, by a further monitoring system (which may or may not be intact).

However, having accepted such mental events as irreducible, and subject to monitoring systems, how should we classify these events? It seems straightforward to classify physical actions (and the preceding neural activity within the motor cortex) as 'acts', subject to the constraints outlined above. It seems less easy, but possible, to classify thoughts as 'acts'; we are given a helpful quotation from Piaget: "thought is internalised action". However, it does not seem possible to classify percepts (or hallucinations), beliefs (or delusions), and emotions as actions. Drs 